\title{
Scintillation efficiency for low energy nuclear recoils in liquid xenon dark matter detectors
}

\author{
Wei $\mathrm{Mu}^{\mathrm{a}}$, Xiaonu Xiong ${ }^{\mathrm{b}}$, Xiangdong $\mathrm{Ji}^{\mathrm{a}, \mathrm{b}, \mathrm{c}, *}$ \\ ${ }^{a}$ INPAC, Department of Physics, and Shanghai Key Lab for Particle Physics and \\ Cosmology, Shanghai Jiao Tong University, Shanghai, 200240, P. R. China \\ ${ }^{b}$ Center for High-Energy Physics, Peking University, Beijing, 100080, P. R. China \\ ${ }^{c}$ Maryland Center for Fundamental Physics, University of Maryland, College Park, \\ Maryland 20742, USA
}

\begin{abstract}
We perform a theoretical study of the scintillation efficiency of the low energy region crucial for liquid xenon dark matter detectors. We develop a computer program to simulate the cascading process of the recoiling xenon nucleus in liquid xenon and calculate the nuclear quenching effect due to atomic collisions. We use the electronic stopping power extrapolated from experimental data to the low energy region, and take into account the effects of electron escape from electron-ion pair recombination using the generalized Thomas-Imel model fitted to scintillation data. Our result agrees well with the experiments from neutron scattering and vanishes rapidly as the recoil energy drops below $3 \mathrm{keV}$.
\end{abstract}

Keywords: Liquid Xenon, Dark Matter, WIMPs, Scintillation Efficiency, Quenching

\section{Introduction}

There is much astrophysical evidence for the existence of the so-called dark matter (DM) in the universe [1]. The most attractive candidate is the weakly interacting massive particle (WIMP) [2, 3, 4]: a neutral stable particle with mass ranging from $\mathrm{GeV}$ to $\mathrm{TeV}$ which undergoes weak interactions. WIMPs can be detected directly by observing the atomic (nuclear) recoils

\footnotetext{
*Email: xdji@sjtu.edu.cn (Xiangdong Ji) 
after they scatter elastically with nuclei through weak interactions. Many direct detection experiments have been proposed and run in the last two decades [5], among which liquid xenon (LXe) experiments have been proven to be particular promising. The recent XENON100 and LUX experiments have yielded the best detection limits in almost all regions of the possible WIMP masses: they are more sensitive than most other experiments using alternative detection media $[6,7]$. Other xenon-type experiments, XMASS, PandaX [8], and XENON1T will soon join the direct search effort.

Analyzing direct-detection data requires reconstructing the nuclear recoil energy through certain calibrations. In LXe experiments, the calibration of the prompt scintillation signal (also called S1) is normally achieved through neutron-induced nuclear recoils. The quantity of interest is the so-called scintillation efficiency, the average amount of the prompt scintillation at a given nuclear recoil energy, which depends on the actual detectors. What is important is the detector independent quantity, relative scintillation efficiency [6],

$$
\mathcal{L}_{\mathrm{eff}}\left(E_{\mathrm{nr}}\right)=\frac{S_{\mathrm{ee}}(\mathcal{E})}{S_{\mathrm{nr}}(\mathcal{E})} \frac{S 1\left(E_{\mathrm{nr}}, \mathcal{E}\right)}{E_{\mathrm{nr}}} \frac{1}{L_{y}(\mathcal{E})},
$$

where $S 1\left(E_{\mathrm{nr}}, \mathcal{E}\right)$ is the scintillation signal from a nuclear recoil with initial energy $E_{\mathrm{nr}}$ while $L_{y}$ is the light yield per $\mathrm{keV}$ from $122 \mathrm{keV}$ electron recoils $\left({ }^{57} \mathrm{Co} \gamma\right.$ calibration line used as a reference energy) in a given drift electric field $\mathcal{E}$. The coefficient $S_{\mathrm{ee}} / S_{\mathrm{nr}}$ corrects for the effect of the drift field applied to the detector so that $\mathcal{L}_{\text {eff }}$ is the relative scintillation at zero drift field $\mathcal{E}=0$. In a LXe detector, nuclear recoils and electron recoils are two different types of interactions types which give rise to two distinct types of signals.

A series of experiments $[9,10,11,12]$ have been carried out to measure $\mathcal{L}_{\text {eff }}$ using the neutron sources. For very low energy nuclear recoils, measurements are particularly difficult. In real applications, $\mathcal{L}_{\text {eff }}$ has to be extrapolated to lower energy from the lowest measured energy $3 \mathrm{keV}$ [11] using various assumptions. However, if the mass of WIMP is around $10 \mathrm{GeV}$, most of the recoiling events will be $2 \mathrm{keV}$ or less $[13,14,15]$ and considerable uncertainties exist in converting experimental data to actual detection limits. Therefore, theoretical investigations of $\mathcal{L}_{\text {eff }}$ are important alternatives. Following a nuclear recoil, the nuclear kinetic energy is transferred to electronic excitations and the thermal motion of the neighboring atoms. A common practice in the literature is to use the Lindhard factor (nuclear quenching 
factor) $[16]$

$$
q_{\mathrm{nc}}\left(E_{\mathrm{nr}}\right)=\eta\left(E_{\mathrm{nr}}\right) / E_{\mathrm{nr}}
$$

to characterize the fraction of the recoil energy given to electronic excitations $\eta\left(E_{\mathrm{nr}}\right)$, and then calculate $\mathcal{L}_{\text {eff }}[17,18,19,20]$. Note that electronic excitations $\eta\left(E_{\mathrm{nr}}\right)$ is a quantity difficult to measure directly. Simulation programs, such as TRIM [21] (TRansport of Ions in Matter) or GEANT4 [22] (GEometry ANd Tracking), can also be used to calculate $q_{\mathrm{nc}}$ in a medium. However, they have not been calibrated by any means for low energy recoils either. For example, a cut-off energy for atoms participating in the cascade is often imposed independent of the low energy behavior of the electronic stopping power. Such cut-offs have little effect on the the nuclear quenching at high-energy, but can change the result significantly at low recoil energy.

In this work, we aim to develop a theoretical approach to simulate the stopping process of a recoiling xenon atom in a LXe medium and get a realistic $q_{\text {nc }}$ in the low energy region, which we argue has been over-estimated in the past. For the input electronic stopping power (ESP), we use experimental data between $40 \mathrm{keV}$ and $100 \mathrm{keV}$ [23] to derive a fit for ESP and then extrapolate it to the low energy region. In calculating the actual scintillation, we consider electrons escape from electron-ion pair recombination by extending the Thomas-Imel model [24] to zero electric field, and fit to the experimental linear-energy-transfer (LET, $=$ ESP divided by density) dependence in LXe to find the free parameters. Combining the above, we obtain an estimate of the (relative) scintillation efficiency at the keV-recoil energy region. The result compares favorably with existing data from neutron scattering and indicates a rapidly vanishing scintillation from the nuclear recoils below $3 \mathrm{keV}$.

This paper is organized as follows: In Section 2, we review the physical stopping process of a recoiling xenon and present our calculation steps, including how to calculate the nuclear energy transfer and the electronic energy dissipation, how to simulate the LXe medium, how to identify the cut-off energy and what ESP we are using. In Section 3, we show how the scintillation yield and quenching are generated and our generalization for Thomas-Imel model which can evaluate the main scintillation quenching effect in the low energy region. In Section 4, we present the results, which provide the trend of the scintillation efficiency in the low energy region. 


\section{A Nuclear Quenching Study At Low-Energy}

In this section, we review current theoretical results for the nuclear quenching effect and present new results for the nuclear quenching factor. We follow the traditional approaches of cascading simulations in the liquid xenon medium, but alter the cascading energy cut-off and the electron stopping power estimate.

\subsection{Theoretical Considerations for the Nuclear Quenching Effect}

Unlike electron recoils, whose energies are almost entirely dissipated into electrons of the medium, the energy dissipation process of nuclear recoils is more complicated. When a xenon nucleus is scattered by a DM particle in the LXe medium, it becomes a recoiling xenon atom (or nucleus) with kinetic energy $E_{\mathrm{nr}}$. After the recoiling xenon atom is thermalized inside the LXe medium, a fraction of the recoil energy is given to the electrons in the medium $\left(\eta\left(E_{\mathrm{nr}}\right)\right)$, the remaining energy is given to the neighboring atoms in the form of heat $\left(E_{\mathrm{nr}}-\eta\left(E_{\mathrm{nr}}\right)\right)$. The energy given to the electrons $\eta\left(E_{\mathrm{nr}}\right)$ is considered to be equivalent to the energy of electron recoils. Naively, the nuclear recoils with energy $E_{\mathrm{nr}}$ will cause the same electron effect (electron ionization or excitation) as that caused by the electron recoils with energy $E_{\text {er }}=\eta\left(E_{\text {nr }}\right)$. To calculate the scintillation efficiency, one can first estimate what fraction of the recoil energy is transferred to the electrons, $q_{\mathrm{nc}}$, which is the so-called Lindhard factor or nuclear quenching factor.

Normally, authors estimate the nuclear quenching factor $q_{\text {nc }}$ through some theoretical calculations, such as Lindhard's basic integral equation [16], or computer simulation programs. Because of the different treatments of the collision cascade process, such as different choice for ESP or cut-off energy, the nuclear quenching factor $q_{\mathrm{nc}}$ from different work varies, and is controversial. For example, the most cited nuclear quenching factor derived from Lindhard's basic integral equation can be expressed by the following equation [25],

$$
q_{\mathrm{nc}}\left(E_{\mathrm{nr}}\right)=\frac{\eta\left(E_{\mathrm{nr}}\right)}{E_{\mathrm{nr}}}=\frac{k g(\epsilon)}{1+k g(\epsilon)},
$$

where $g(\epsilon)$ is an empirical expression which can be found in Ref. [25] and $k$ is the proportionality constant between the $\operatorname{ESP}(d E / d x)_{\text {el }}$ and the velocity of the projectile (recoil atom) [26]. In this expression, the parameter $k$ is controversial. For xenon, there are two widely cited values: $k=0.166$ proposed by Lindhard in Ref. [16] and $k=0.110$ recalculated by Hitachi in Ref. [17]. 
One can also calculate the nuclear quenching factor $q_{\text {nc }}$ from computer simulations. We use TRIM from the SRIM-2013 package [21] to simulate the stopping process of an energetic xenon atom in xenon medium. From this result, we get the equivalent electron recoils energy $\eta\left(E_{\mathrm{nr}}\right)$ and obtain the nuclear quenching factor $q_{\mathrm{nc}}$. Although much study exists for the quenching factor, different treatments do not agree with each other. In fact, the most quoted Lindhard factors or computer simulation results are scattered as shown in Fig. 1.

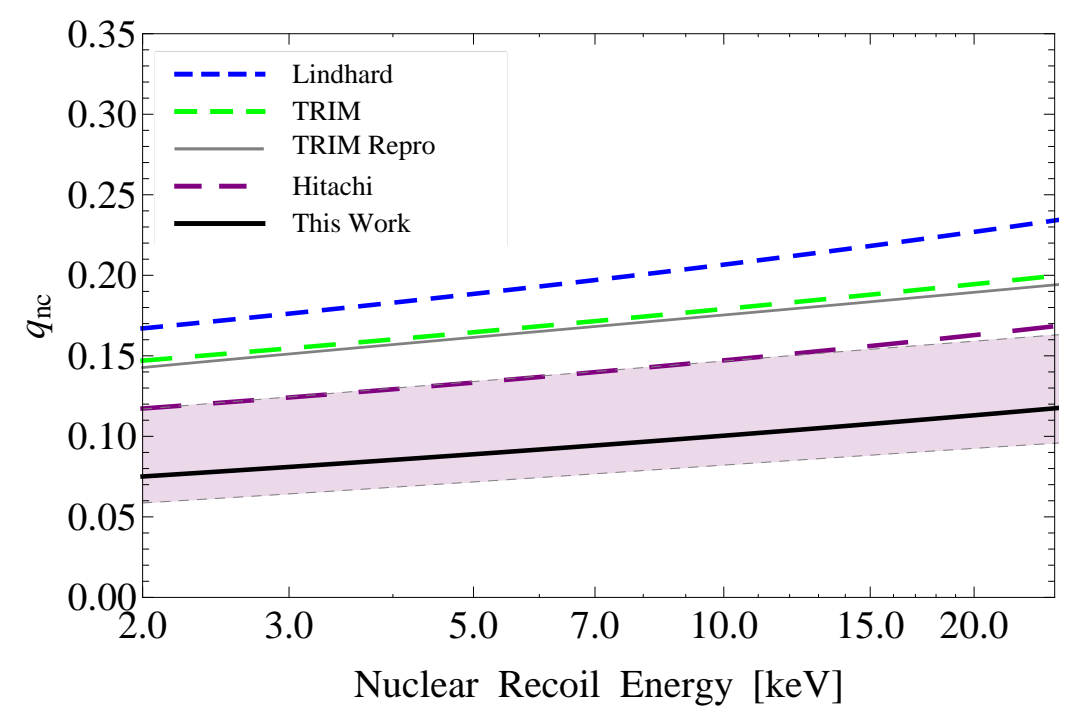

Figure 1: The nuclear quenching factor $q_{\mathrm{nc}}$ of LXe obtained from different theoretical predictions. The lower black solid curve is the result from our calculation by using the ESP fitted to available experimental data.

Because theoretical estimates of the nuclear quenching at low-energy differs considerably and many of the approximations are not justifiable in this region, the existing theoretical results for the nuclear quenching factor are un-reliable. In the following section, we perform a more realistic analysis by re-examining the cascading process and the theoretical input for the electronic stopping power.

\subsection{Cascading Simulation}

The stopping process of the nuclear recoils is in principle a many-body quantum mechanics problem, and we must make reasonable simplifications 
to render a theoretical treatment feasible. Similar to previous works, we treat the nucleus-nucleus interactions classically as binary elastic collisions, since their de Broglie wavelengths (less than $0.0025 \AA$ ) in the concerned energy region $(0.5 \sim 25 \mathrm{keV})$ are much smaller than the atomic scale. Meanwhile, the nucleus-electron interaction is treated as a small "friction" on the projectile along the nucleus trajectory. This picture is justified because the atomic scattering generates large momentum transfer, and hence influences the atom trajectory without dissipating kinetic energy, whereas the atom-electron interaction generates little momentum transfer but appreciable kinetic energy dissipation, which can be considered as a small perturbation to atomic motion.

When considering the energy transfer in binary atomic collisions, we use the classical elastic scattering approximation. Elastic scattering slows down the recoiling atom through momentum transfer to the target, both of which become the secondary recoiling atoms that trigger a collision cascade. Based on the binary collision kinematics, we have the energy transfer to the target in a collision [27]

$$
T=E_{0} \sin ^{2}\left(\theta_{c} / 2\right),
$$

where $T$ and $E_{0}$ is the transferred energy and projectile energy of the initial nucleus in lab frame. The scattering angle in the center-of-mass frame $\theta_{c}$ can be derived from the following expression [27]

$$
\theta_{c}=\pi-2 b \int_{r_{0}}^{\infty} \frac{1}{r^{2} \sqrt{1-U(r) / E_{c}-b^{2} / r^{2}}} d r
$$

where $b$ is the impact parameter and $E_{c}$ is the center-of-mass energy. The lower limit $r_{0}$ is the nearest distance between the projectile and target nuclei determined by the following equation

$$
1-\frac{U(r)}{E_{c}}-\frac{b^{2}}{r_{0}^{2}}=0 \text {. }
$$

We choose the screened Coulomb potential for the inter-atomic interaction,

$$
U(r)=\frac{Z^{2} e^{2}}{r} \Phi\left(\frac{r}{a}\right)
$$

where $\Phi\left(\frac{r}{a}\right)$ is the Hartree-Fock screening function and $a=\frac{1}{2}\left(\frac{3 \pi}{4}\right)^{2 / 3} \frac{a_{0}}{2 Z^{0.23}}$ is the Hartree-Fock screening radius for xenon ( $a_{0}$ is the Bohr radius). The 
empirical expression for the screening function is [28],

$$
\Phi(x)=0.1818 e^{-3.2 x}+0.5099 e^{-0.9423 x}+0.2802 e^{-0.4028 x}+0.02817 e^{-0.2016 x} .
$$

In our simulation, $b$ is randomly generated according to the spatial distribution of xenon atoms as we will explain latter. The scattering angle $\theta_{c}$ and the energy transferred to the target are calculated through Eq. (5) and Eq. (4).

When the projectile is approaching the target, the trajectory is curved by the screened Coulomb potential between the projectile and the target. Therefore, the flight path of the recoiling atom between two successive collisions follows the slightly curved trajectory, connecting two points: the initial position of the projectile and the ending position where the projectile and the target nuclei are closest. The length of the flight path is a curvilinear integral from the two atoms' initial distance $r=R$ to their closest point $r=r_{0}$ along the trajectory

$$
\lambda=\int_{r_{0}}^{R} \sqrt{d r^{2}+r^{2}(d \chi)^{2}},
$$

where $\chi=\theta_{c} / 2$ is the polar angle. Applying the trajectory equation from Eq. (5), $\lambda$ can be written as

$$
\lambda=\int_{r_{0}}^{R} d r \sqrt{1+\frac{b^{2}}{r^{2}\left(1-U(r) / E_{c}-b^{2} / r^{2}\right)}} .
$$

Then the energy given to electrons in one nuclear binary collision is determined by,

$$
\eta\left(E_{\mathrm{nr}}^{i}\right)=\int_{0}^{\lambda\left(E_{\mathrm{nr}}^{i}\right)} S_{e} d x
$$

where $E_{\mathrm{nr}}^{i}$ is the projectile energy of the nucleus in the concerned binary collision and $S_{e}$ is the ESP, the energy loss to the electrons per unit path $(d E / d x)_{\mathrm{el}}$. Summing $E_{\mathrm{nr}}^{i}$ up through the whole cascade, we obtain the recoil energy given to electrons $\eta\left(E_{\mathrm{nr}}\right)$.

In some simulation programs like GEANT4 and TRIM, the medium is treated as homogeneous in which the atoms are randomly distributed according to a uniform probability distribution. Here we consider a more realistic spatial distribution of nuclei in LXe. The recoiling behavior in LXe depends strongly on the positions of neighboring atoms, so a realistic probability distribution at small distance scales is important for determining the free flight 
paths and the impact parameters. For a homogeneous medium like LXe, the spatial distribution of the atoms is characterized by the radial distribution function, also called the pair correlation function (PCF) $g(r)$, which is defined as the probability of finding a particle at distance $r$ from a reference particle. Generally speaking, the pair correlation function is determined by the inter-atomic interactions and the environmental temperature. The function can be calculated theoretically using molecular dynamic simulations or measured experimentally in neutron diffraction. We take $g(r)$ from Ref. [29] in this work.

Considering a xenon atom which gains an initial momentum from either a DM particle or another recoiling xenon nucleus, it then becomes a projectile atom which will undergo further binary collisions. The target xenon atom participating in the subsequent collision can be selected from the 5 to 6 nearest neighbors of the projectile in the forward direction. We can simulate the nearest neighbor collision by two parameters: a distance $(R)$ to the projectile atom and an angle $(\Theta)$ between the radial and projectile moving directions. The distance $R$ is distributed according to the PCF $g(r)$, and generated using an acceptance-rejection method. The angle $\Theta$ is distributed in a way that makes the atoms isotropically distributed. Note, for an individual binary atomic collision, the angles $\Theta$ should also relate to the spatial distribution of the neighbors. However, in a large number of binary atomic collisions, the angles are expected to satisfy above distribution due to the isotropic nature of the liquid. Once a pair of $R$ and $\Theta$ is determined, we calculate the impact parameter as $b=R \sin \Theta$. After the nearest neighbors are generated, we choose the one whose distance to the projectile nucleus is smallest as the target for the following binary atomic collision.

When the projectile nucleus is scattered, its kinetic energy is transferred partially to the target and the moving direction is changed. The two nuclei will be regarded as new projectile particles and further scattered by the other target nuclei, and so on. During the binary atomic collisions, the electrons-nuclei interaction is treated as a small friction, which results in energy dissipation from the projectile to the electrons of its neighbors [30] and the energy dissipation is described by the ESP. We generally ignore the charge state of the atoms by assuming all atoms interact with a screened potential described previously. Transferring energy to other nuclei and dissipating energy to electrons, the moving particle slows down and the trajectory will be terminated when its kinetic energy approaches a predefined cut-off energy. 


\subsection{Cascading Energy Cut-off}

The cut-off energy or the threshold energy is an energy below which the energy transfer from the moving particle to atomic electrons is forbidden. Thus below the cut-off energy, all energy transfer is in the form of phonons (heat). With other parameters fixed, the larger the cut-off energy is set in the simulation, the smaller the equivalent electron recoils energy $\eta\left(E_{\mathrm{nr}}\right)$ will be, since more energy is converted to phonons. This cut-off energy in a sense simulates the minimal energy cut-off discussed in Ref. [31], which is a quantum mechanical effect.

Ficenec et al. have discussed the quantum effect in their research on electronic excitation by extremely slow protons in Ref. [32]. In that work, the authors estimate the threshold energy using simple two-body kinematics, and assume that energy transfer from the ionizing particle to an atomic electron is forbidden if the minimum excitation energy of the system $E_{g}$ exceeds the maximum possible energy transfer to the electron. Based on this assumption, they derive a threshold velocity for the moving particle $v_{t h}=E_{s} / 2 m_{e} v_{F}$, where $m_{e}$ is the electron mass and $v_{F}$ is the Fermi velocity of the atomic electrons. Identifying $E_{g}$ with the gap energy in LXe $(9.3 \mathrm{eV})$, the cut-off energy is calculated as $20 \sim 40 \mathrm{keV}$.

However, experimental measurements have shown that this type of estimation does not work, although a downward trend in the scintillation starts to appear at that energy. In Ref. [32], the authors also notice that even extremely slow protons with velocity far below the calculated threshold velocity can excite atomic electrons. The XENON100 experiment has observed scintillation and ionization when the recoil xenon energy is as low as $3 \mathrm{keV}$. Theoretical arguments indicate that classical processes with a continuous energy transfer can be brought to reconciliation with the quantum mechanical mechanism of discrete energy transfer through some statistical sense [33]. In our case, the kinematic cut-off shall be reflected through a fast fall-off of the ESP at low energy, which vanishes entirely below the gap energy. As we shall see, since our model for the simulation will not include this artificial cut-off energy, we take care of the kinematic cut-off effect using the following criteria instead: if the kinetic energy of the initial projectile xenon is lower than the gap energy in LXe $(9.3 \mathrm{eV})$, we will not initiate the collision cascade since no electronic excitations or ionizations can be produced; and we will terminate a trajectory when the kinetic energy of the moving particle is approaching the thermal energy of atoms in LXe $(0.02 \mathrm{eV})$. 


\subsection{A New Empirical ESP}

Another key parameter for the simulation is the ESP of xenon in the low energy region. In principle, the ESP as a function of the projectile's kinetic energy can be measured experimentally. However, there is little data in the low energy region of our interest $(\sim \mathrm{keV})$. We have to rely on theoretical considerations and limited data at moderate energies to make progress. Theoretical studies of the ESP for a charged particle moving in media has been of great interest for over a century [34]. Due to complicated physical processes, theoretical results are scattered. Both the Lindhard-Scharff (L-S) theory [35] and Brandt-Kitagawa (B-K) theory [36] predicted ESP with linear dependence on velocity $v$. However, Tilinin's study shows that the stopping power decreases much faster, like $v^{3}$ [37]. Ziegler et al. [21] proposed a semiempirical result and summarized in a Stopping/Range Table, which is also proportional to velocity at low energy. As shown in Fig. 2, the SRIM data is substantially lower than the predictions of L-S and B-K theories, but higher than that of Tilinin.

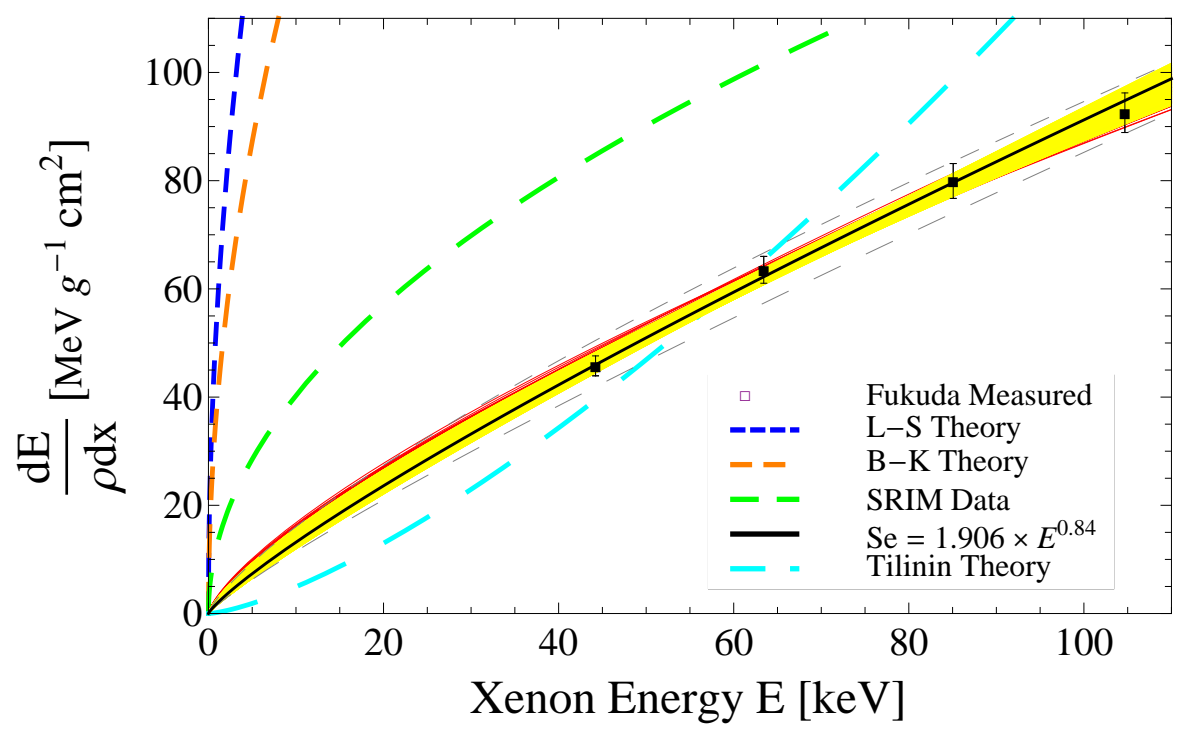

Figure 2: Comparisons between different theoretical predictions [21, 35, 36, 37] and experimental data [23] on the ESP for a xenon atom in LXe. The ESP fitted from Fukuda's measured data is shown as the solid line and the gray dashed curve bond the $2 \sigma$ error region (yellow-colored band). 
We have also shown the available experimental data in Fig. 2, which was measured in gas phase using low energy $(40 \sim 200 \mathrm{keV}) \mathrm{Xe}^{+}$[23]. Since the Van der Waals forces are relatively weak for noble gases, the ESP in the liquid phase will be close to that in the gas phase [28]. In this work, we ignore the charge states of the collision atoms and the phase effect of the ESP in our simulation. The data indicates a faster fall-off at lower energy than $v$, but not as faster as Tilinin's. Assuming the ESP drops following the same trend in the concerned energy region as that measured at higher energy with a power law behavior $E^{\alpha}$, we fit the power law exponent and the coefficient to the lowest four measured points, and extrapolate the result to the low energy region. The best fit yields,

$$
S_{e}(E)=1.906 \times(E / \mathrm{keV})^{0.84} \mathrm{MeV} \cdot \mathrm{g}^{-1} \cdot \mathrm{cm}^{2} .
$$

The result is also shown in Fig. 2 as the black solid line, which lies in between Ziegler's result and Tilinin's. As an estimate of the uncertainty, we also generate the $2 \sigma$ error region by randomly generating series ESP functions based on the variations of Fukuda's datapoints and using $\chi^{2}$ distribution to pick out the ones within the $2 \sigma$ error region of the datapoints (yellow-colored region in Fig. 2). Then we determine a range of extrapolations defined by two boundary curves, $S_{e}^{\prime}(E)=2.729 \times E^{0.77}$ and $S_{e}^{\prime \prime}(E)=1.551 \times E^{0.87}$, which confine the $2 \sigma$ region of the data, and are shown as dashed gray lines in Fig. 2.

Using the above result, we calculate $q_{\text {nc }}$ through Monte Carlo simulations. Between $0.5 \mathrm{keV}$ and $25 \mathrm{keV}$, we choose a discrete set of recoil energies and simulate 10,000 scattering events for each energy. We get a fair average nuclear quenching factor for the given recoil energy and derive a phenomenological fit for the energy range $0.5 \sim 25 \mathrm{keV}:$ :

$$
q_{\mathrm{nc}}\left(E_{\mathrm{nr}}\right)=\frac{e^{-0.033 E_{\mathrm{nr}}^{-0.958}}}{1+13.789 E_{\mathrm{nr}}^{-0.189}} .
$$

We plot it in Fig. 1 as the black solid line. We have also shown the $2 \sigma$ band calculated with the $2 \sigma$ fit of the ESP as the shaded band. To calibrate our code, we perform simulations using the SRIM ESP with TRIM parameters, such as the equivalent cut-off energy, in attempt to reproduce the TRIM results. We plot the original TRIM result as the green dashed curve and our reproduction in gray solid curve. The small difference is due to our use of a realistic atomic distribution in LXe. We also plot the widely used 
Lindhard factor [18] as the blue and purple dashed curve for comparison, using $k=0.166$ and $k=0.110$ separately.

Obviously, our $q_{\mathrm{nc}}$ is smaller than the quoted Lindhard factor or the TRIM result, which is mainly caused by the smaller ESP used in our simulation. The theoretical ESPs are varied up to an order of magnitude, but the difference between the nuclear quenching factors is not so significant as that between the corresponding ESPs. This is caused by the different cut-off energy (or equivalent cut-off energy) used in different treatments. For example, we use the natural cut-off energy (the thermal energy of LXe) in our simulation while TRIM uses some artificial cut-off energy such as the displacement energy which is large compared with ours. In the meanwhile, the natural cut-off energy results in the fact that the small error band around the ESP is translated into a broad band in the nuclear quenching factor. Since the thermal energy of LXe is very small $(0.02 \mathrm{eV})$, many secondary nuclear recoils whose energy is very small are involved in the collision cascade process. If we only replace the ESP with that from SRIM or Lindhard's theory in our code, the nuclear quenching factor will be much larger than the results from TRIM or Eq. (3).

\section{Recombination of Electron-Ion Pairs}

After all recoiling atoms are thermalized, the nuclear recoils, similar to electron recoils, produce a certain number of excitons $\left(N_{e x}\right)$ and electron-ion pairs $\left(N_{i}\right)$, which can be described using Platzman's theory [38]:

$$
\eta\left(E_{\mathrm{nr}}\right)=N_{i} E_{i}+N_{e x} E_{e x}+N_{i} \epsilon
$$

where $E_{i}$ and $E_{e x}$ are the average energy to produce one electron-ion pair and one exciton, and $\epsilon$ is the average kinetic energy of sub-excitation electrons. The excitons' relaxation and the electron-ion pairs' recombination will yield scintillation signals [39].

However, some electrons may escape from recombination even under zero electric field, and only a fraction $r$ of electrons are recombined with ions. This is true for both electron recoils and nuclear recoils and must be taken into account when calculating $\mathcal{L}_{\text {eff }}$. Assuming a fraction $r$ of the electron-ion pair get recombined, we can derive:

$$
\mathrm{S} 1=\mathcal{K} \times\left(N_{e x}+r \times N_{i}\right)
$$


where $\mathcal{K}$ is the coefficient between the number of excitons and the S1 signal. For the same detector, $\mathcal{K}$ for electron recoils should be the same as for nuclear recoils.

Substituting Eq. (2) and Eq. (15) into Eq. (1) and setting the drift electric field $\mathcal{E}=0$, we obtain the expression for $\mathcal{L}_{\text {eff }}$ :

$$
\mathcal{L}_{\text {eff }}\left(E_{\mathrm{nr}}\right)=q_{\mathrm{nc}}\left(E_{\mathrm{nr}}\right) \times \frac{\frac{N_{e x}\left(\eta\left(E_{\mathrm{nr}}\right)\right)+r_{\mathrm{nr}} \times N_{i}\left(\eta\left(E_{\mathrm{nr}}\right)\right)}{\eta\left(E_{\mathrm{nr}}\right)}}{\frac{N_{e x}\left(E_{\gamma}\right)+r_{\gamma} \times N_{i}\left(E_{\gamma}\right)}{E_{\gamma}}} .
$$

where $E_{\gamma}=122 \mathrm{keV}$ and we distinguish fraction $r$ for nuclear recoils and electron recoils as $r_{\mathrm{nr}}$ and $r_{\gamma}$, which may be different. Once the recombination probability $r$ and the ratio $N_{e x} / N_{i}$ are determined, $\mathcal{L}_{\text {eff }}$ for a particular nuclear recoil energy can be calculated.

The Thomas and Imel box model was originally proposed to explain the electric field dependence of electron-ion recombination in liquid argon or xenon for electron recoils [24]. Through various generalizations, this model successfully describes the electron-ion pair recombination in LXe for nuclear recoils $[19,26,40]$. In this model, the electron-ion recombination rate depends on the external electric field $\mathcal{E}$ as well as the free-charge density $N_{0}$,

$$
r=1-\frac{N_{i}^{\mathrm{esc}}}{N_{i}}=1-\frac{1}{\xi} \ln (1+\xi) \quad \xi=\frac{N_{0} \alpha}{4 a^{2} \mu_{-} \mathcal{E}},
$$

where $\alpha /\left(4 a^{2} \mu_{-}\right)$is a constant determined by the recombination coefficient $\alpha$, the electron mobility coefficient $\mu_{-}$, and the ionization-volume length scale $a$. However, when there is no applied field, we must consider the diffusion term of the free electrons. Diffusion can be modelled as a constant background field, which cause some electrons to escape recombination. Extending the model to zero external field, $\xi$ depends only on the ion density $N_{0}$ in the localized region, which should be linearly proportional to LET at low energy. Hence we define $\xi \equiv K \times$ LET, where $K$ is a free parameter obtained phenomenologically from the LET dependence of the scintillation yields in LXe $[41,42]$.

The value for $N_{e x} / N_{i}$ varies from 0.06 to 0.2 for LXe [38, 39, 42, 43] for electron recoils, where 0.06 is the theoretical value [43] and 0.2 the measured one [42]. In Ref. [44], $N_{e x} / N_{i}=1.05$ or 1.09 is claimed for nuclear recoils from the direct charge measurement. Note, these values are fitted to the data based on a much larger $q_{\mathrm{nc}}$ in Ref. $[17,18]$. If we used the quenching factor 
advocated here and redo the fitting, we find $N_{e x} / N_{i}$ can be in the range of 0.1 to 0.3 . Therefore, the ratios $N_{e x} / N_{i}$ for the electron and nuclear recoils seem similar if regarding the energy $\eta\left(E_{\mathrm{nr}}\right)$ as equivalent electron recoils energy, despite that the ion densities in both cases are quite different. In the following, we assume this is true and take $N_{e x} / N_{i}=0.20$ to fit the free parameter $K$ to the experimental measured data in Ref. [42]:

- $K=2.53 \mathrm{MeV}^{-1} \cdot \mathrm{g} \cdot \mathrm{cm}^{-2}$

Based on the above parameter, we show in Fig. 3 the LET dependence of the relative xenon scintillation yields to electronic recoils at $122 \mathrm{keV}$.

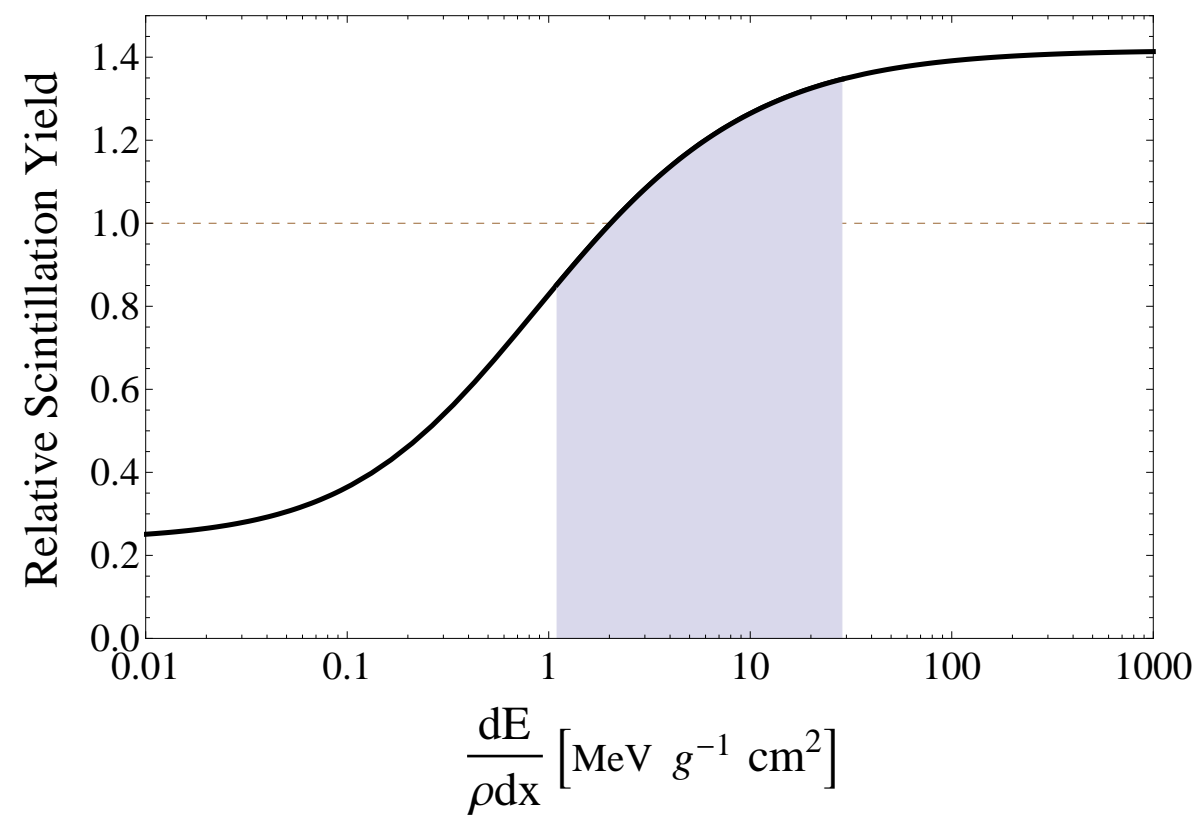

Figure 3: LET dependence of the relative scintillation yields to electronic recoils at $122 \mathrm{keV}, \frac{S 1\left(E_{\mathrm{nr}}\right) / \eta\left(E_{\mathrm{nr}}\right)}{L_{y}}$, in liquid xenon from Thomas-Imel model. The black solid curve, which we consider as the best fit, corresponds to the free parameters set to $K=2.53 \mathrm{MeV}^{-1} \cdot \mathrm{g} \cdot \mathrm{cm}^{-2}$ and $N_{e x} / N_{i}=0.20$. The shaded region corresponds to the interest of direct DM detection (LET for $0.5 \mathrm{keV}$ to $25 \mathrm{keV}$ nuclear recoils energy).

Since the LET dependence of the scintillation yields in LXe has been observed for both electron and heavy incident particles in LXe, we can predict the electron-ion pairs recombination rate from the Thomas-Imel model for 
both nuclear recoils and $122 \mathrm{keV}$ electron recoils. Based on the predictions from Eq. (17), the recombination rate for $122 \mathrm{keV}$ electron recoils is less than for nuclear recoils if the nuclear recoils energy $E_{\mathrm{nr}}$ is larger than $1 \mathrm{keV}$. This is explicitly shown in in Fig. 3 that the relative (to electron recoils) scintillation yield of nuclear recoils is larger than 1 .

\section{Comparison with Data and Discussion}

To get the result for $\mathcal{L}_{\text {eff }}$, we substitute the nuclear quenching factor for nuclear recoils in Eq. (13), the recombination rate $r_{\mathrm{nr}}$ for nuclear recoils and $r_{\gamma}$ for electron recoils from Eq. (17) into Eq. (16). The final result for $\mathcal{L}_{\text {eff }}$ shown as the solid line in Fig. 4 and is phenomenologically expressed as:

$$
\mathcal{L}_{\mathrm{eff}}\left(E_{\mathrm{nr}}\right)=q_{\mathrm{nc}}\left(E_{\mathrm{nr}}\right) \times \frac{1.417-0.245 \ln \left(1+4.822 E_{\mathrm{nr}}^{0.840}\right)}{E_{\mathrm{nr}}^{-0.840}} .
$$

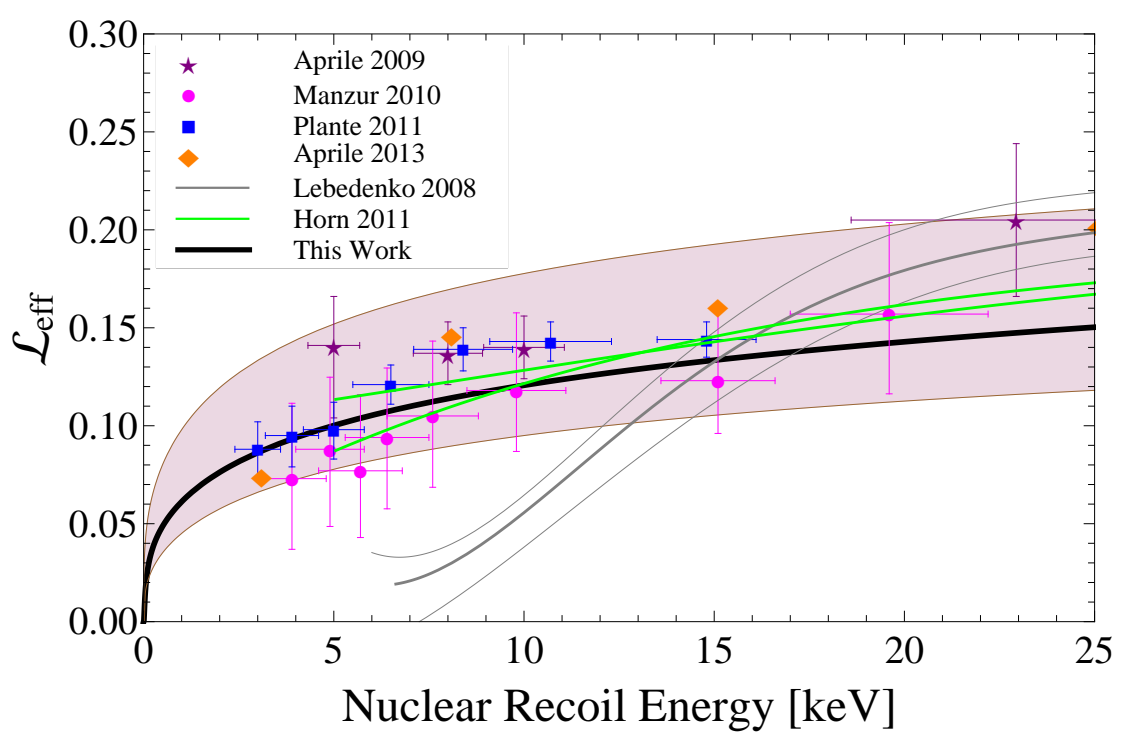

Figure 4: The relative scintillation efficiency $\mathcal{L}_{\text {eff }}$ obtained from our calculation, compared with the available experimental data $[9,10,11,12,45,46]$. The shaded band shows the system and statistical uncertainties with $\pm 2 \sigma$.

The error band includes both the systematic $2 \sigma$ uncertainties from the ESP fit and the statistical $2 \sigma$ uncertainties in the simulation. Along with our 
calculation, we have also shown experimental data from different measurements. The blue squares are from the Columbia group measurement [9]. The magenta dots are from the Yale group measurement [10]. The purple stars and the orange diamonds are from the XENON100 collaboration [11, 12]. The green and gray solid line are from ZEPLIN-III collaboration [45, 46]. Most notably, the scintillation efficiency rapidly decreases with decreasing energy, particularly when less than $3 \mathrm{keV}$ where no experimental data exist, and goes to zero as the energy goes to zero. This result is expected to be generally valid since as the recoil energy becomes small, the atom loses kinetic energy mainly through elastic scattering with other atoms, rather than through electronic ionizations or excitations. This behavior has been in some sense already encoded in the ESP.

To conclude, we have formulated a more realistic picture of scintillation efficiency for the low energy nuclear recoils in LXe. Compared with previous theoretical analyses, such as the results in Ref. $[18,19]$, we re-calculate the nuclear quenching factor using Monte-Carlo simulation together with a fast fall-off ESP and natural cut-off energy. In addition, we study the LET dependence of scintillation yields in LXe to predict the electron-ion recombination effect. The study is not completely ab initio, but it is the most realistic and comprehensive treatment at this time. Our results compare favorably with the data from neutron scattering, but differ somewhat from the Monte Carlo fitting to the broad-spectrum neutron data [45, 46, 47]. The results indicate that LXe scintillation response drops very quickly below $3 \mathrm{keV}$, which is a general feature that is independent of many details of the study. We have also used the above model including the electric field dependence to study the ionization yield, which can potentially be used either independently or with scintillation together to determine the energy scale of the nuclear recoils [48].

\section{Acknowledgment}

W. M. thanks Prof. James F. Ziegler for helpful suggestions and discussions. This work is partially supported by a 973 project, No. 2010CB833005, of China's Ministry of Science and Technology, and a grant (No. 11DZ2260700) from the Office of Science and Technology in Shanghai Municipal Government. This work has also been supported by the U.S. Department of Energy via grant DE-AC02-05CH11231. 


\section{References}

[1] G. Bertone, D. Hooper and J. Silk, Phys. Rept. 405, 279 (2005)

[2] M. W. Goodman and E. Witten, Phys. Rev. D 31.123059 (1985)

[3] G. Goodman, M. Kamionkowski and K. Griest, Phys. Rep. 267.5 195 (1996)

[4] W. L. Freedman and M. S. Turner, Phys. Rev. Phys. 751433 (2003)

[5] D. Akimov, Nucl. Instrum. Meth. A 628, 50 (2011).

[6] E. Aprile et al. [XENON100 Collaboration], Phys. Rev. Lett. 109, 181301 (2012)

[7] D. S. Akerib et al., [arXiv:1310.8214 [astro-ph.CO] (2013).

[8] X. G. Cao et al., [arXiv:1405.2882 [physics.ins-det]] (2014).

[9] E. Aprile et al., Phys. Rev. C 79, 045807 (2009)

[10] A. Manzur et al., Phys. Rev. C 81, 025808 (2010)

[11] G. Plante et al., Phys. Rev. C 84, 045805 (2011)

[12] E. Aprile et al. [XENON100 Collaboration], Phys. Rev. D 88.1, 012006 (2013)

[13] R. Bernabei et al. [DAMA Collaboration], Eur. Phys. J. C 56, 333 (2008)

[14] C. E. Aalseth et al. [CoGeNT Collaboration], Phys. Rev. Lett. 106, 131301 (2011)

[15] R. Agnese et al. [CDMS Collaboration], [arXiv:1304.4279 [hep-ex]].

[16] J. Lindhard et al., Kgl. Danske Videnskab., Selskab. Mat. Fys. Medd. 33 10, (1963)

[17] A. Hitachi, Astropart. Phys. 24, 247 (2005)

[18] D. -M. Mei, et al. Astropart. Phys. 30, 12 (2008)

[19] F. Bezrukov, et al. Astropart. Phys. 35, 119 (2011) 
[20] M. Szydagis, et al. JINST 6.10, P10002 (2011)

[21] J. F. Ziegler, http://www.srim.org/

[22] S. Agostinelliae, J. Allision et al., Nucl. Instrum. and Meth. A 506, 250 (2003).

[23] A. Fukuda, J. Phys. B: At. Mol. Opt. Phys 14, 4533 (1981)

[24] J. Thomas and D. A. Imel, Phys. Rev. A 36, 614 (1987)

[25] J. D. Lewin and P. F. Smith, Astropar. Phys. 6, 87 (1996)

[26] P. Sorensen, et al. Phys. Rev. D 83, 063501 (2011)

[27] L. D. Landau and E. M. Lifshitz, Mechanics, Butterworth-Heinemann Ltd Press, Oxford, U.K. (1976)

[28] J. F. Ziegler, et al. Nucl. Instrum. Meth. B 268, 1818 (2010)

[29] K. Wamba, Ph.D thesis, Stanford University (2006) [DOI:oclc/123408748].

[30] J. Eichler, Lectures on ion-atom collisions: From nonrelativistic to relativistic velocities Elsevier Science (2005)

[31] J. I. Collar and D. N. McKinsey, arXiv:1005.0838 [astro-ph.CO].

[32] D. J. Ficenec et al., Phys. Rev. D. 36.1311 (1987)

[33] J. D. Jackson, Classical Electrodynamics, (3rd Edition, John Wiley \& Sons Inc. 1999).

[34] N. Bohr, Philos. Mag. 25, 10 (1913).

[35] J. Lindhard and M. Scharff, Phys. Rev. 124, 128 (1961).

[36] W. Brandt and M. Kitagawa, Phys. Rev. B 25.9, 5631 (1982).

[37] I. S. Tilinin, Phys. Rev. A 51, 3058 (1995);

[38] R. L. Platzman, Int. J. Appl. Radiat. Is. 10, 116 (1961)

[39] E. Aprile and T. Doke, Rev. Mod. Phys. 822053 (2010) 
[40] C. E. Dahl, Ph.D. thesis, Princeton University (2009)

[41] T. Doke et al., Nucl. Instrum. Meth. A 269, 291 (1988)

[42] T. Doke et al., Jpn. J. Appl. Phys. 41, 1538 (2002)

[43] T. Takahashi et al. Phys. Rev. A 12, 1771 (1975)

[44] E. Aprile et al., Phys. Rev. Lett. 107051301 (2011)

[45] V. N. Lebedenko, et al. Phys. Rev. D 80, 052010 (2009)

[46] M. Horn, et al. PHYS. LETT. B 705, 471 (2011)

[47] P. Sorensen, et al. Nucl. Instrum. Meth. A 601, 339 (2009)

[48] W. Mu and X. Ji, [arXiv:1310.2094 [physics.ins-det]]. 